\title{
Surgical procedures for papillary thyroid carcinoma located in the thyroid isthmus: an intention-to- treat analysis
}

This article was published in the following Dove Press journal:

OncoTargets and Therapy

22 August 2016

Number of times this article has been viewed

\author{
Jianyong Lei ${ }^{1,2}$ \\ Jinqiang Zhu' \\ Zhihui Li' \\ Rixiang Gong' \\ Tao Wei'
}

'Thyroid and Parathyroid Surgery Center, ${ }^{2}$ State Key Laboratory of Biotherapy, Collaborative Innovation Center of Biotherapy, West China Hospital, Sichuan University, Chengdu, People's Republic of China
Correspondence: JQ Zhu

Thyroid and Parathyroid Surgery Center, West China Hospital of Sichuan University, 37 Guoxue Xiang, Chengdu 6I004I, People's Republic of China

$\mathrm{Tel} / \mathrm{fax}+862885423822$

Email zjqdoctor@।63.com
Objective: We sought to evaluate and compare the outcomes of different surgical protocols for papillary thyroid cancer (PTC) located in the isthmus in a retrospective intention-to-treat analysis.

Patients and methods: The data of 3,068 patients who received thyroidectomy due to thyroid cancer in our center were reviewed. Of these, 103 patients had a dominant carcinoma located in the isthmus. Various baseline and tumor characteristics and surgical outcomes were evaluated and compared with respect to the different surgical protocols ( 85 cases with total thyroidectomy and 18 cases with less-than-total thyroidectomy). Univariate and multivariate analyses were performed to identify resected patients who developed recurrence with isthmic PTC.

Results: The postoperative complication rates were comparable between the two groups $(17.6 \%$ versus $11.1 \%, P=0.500)$. Although the total thyroidectomy group showed a much higher rate $(P=0.004)$ and number $(P<0.001)$ of parathyroidectomies, long-term follow-up indicated that parathyroid autotransplantation did not significantly damage the function of the parathyroid $(P>0.05)$. Tumor recurrence was observed in five patients, including two patients in the total thyroidectomy group and three patients in the less-than-total thyroidectomy group; the tumor recurrence rate in the total thyroidectomy group was significantly lower than that in the lessthan-total thyroidectomy group $(P=0.040)$. Univariate and multivariate analyses indicated lessthan-total thyroidectomy as a risk factor for tumor recurrence in PTC cases with tumors located at the isthmus (hazard ratio: $1.870,95 \%$ confidence interval: $1.320-2.218, P<0.001$ ).

Conclusion: Our findings indicate that total thyroidectomy is an appropriate initial surgical protocol for isthmic PTC due to the lower recurrence rate, comparable postoperative complication rate, and parathyroid function recovery.

Keywords: papillary thyroid carcinoma, thyroid, isthmus

\section{Introduction}

Thyroid cancer is the most frequent endocrine carcinoma, occurring in $\sim 5 \%-10 \%$ of patients with a thyroid nodule. ${ }^{1}$ The incidence of papillary thyroid cancer (PTC) has increased rapidly in the last two decades, ${ }^{2,3}$ mainly due to the advent of diagnostic procedures such as ultrasonography and ultrasound-guided fine needle aspiration (FNA) biopsy. ${ }^{4,5}$ In particular, the number and thinness of anatomical slices have resulted in a marked increase in the rate of preoperative diagnosis of papillary thyroid microcarcinoma. ${ }^{6}$ Thyroidectomy and ablation by iodine (I)-131 followed by long-term levothyroxine (LT4) suppression of thyrotropin (thyroid stimulating hormone, TSH) is the traditional treatment for differentiated thyroid cancer. ${ }^{7}$ 
The surgical management of thyroid cancer located in the isthmus remains unclear and controversial. Some researchers argue that PTCs located in the isthmus are more likely to be associated with multifocal disease, lymph node involvement, and capsule invasion, thus supporting total thyroidectomy as the first-choice treatment for these patients. ${ }^{8,9}$ However, some opposing conclusions have been proposed; for instance, isthmusectomy alone may be sufficient because this approach does not require exploration of the tracheoesophageal grooves and thus reduces the postoperative complications and operating time. ${ }^{10}$ To our knowledge, there are currently no precise guidelines for the management of patients with dominant thyroid nodules of the isthmus, even according to the American Thyroid Association (ATA) or the European Thyroid Association (ETA). ${ }^{7,11}$

Few reports exist in the literature on thyroid isthmusectomy; most series include only small numbers of malignant lesions, and no consistent conclusions have been drawn. ${ }^{8-10}$ This study was designed to analyze the outcomes (tumor recurrence and postoperative complications) of different surgical protocols. This is the first report from mainland China and one of the largest series reporting on the surgical procedures for PTC confined to the isthmus.

\section{Patients and methods}

A total of 3,068 patients undergoing thyroidectomy due to thyroid cancer during the period between March 2009 and May 2015 in the Department of Thyroid and Parathyroid Surgery Center in West China Hospital of Sichuan University, People's Republic of China, were enrolled. Of these, 103 patients had a dominant thyroid nodule in the isthmus. The inclusion criteria for our study consisted of PTC confirmed by histopathological diagnosis and preoperative ultrasound diagnosis of a nodule located in the thyroid isthmus. The exclusion criteria included a PTC node located in the gland rather than the isthmus, patients who underwent initial treatment elsewhere before referral, those who were considered unresectable at the time of referral, and patients lost to follow-up (two cases), or those (three cases) with missing clinical data that may severely disturb the statistics and the results, with information lost by $>30 \%$. The medical data of patients with a final pathologic report of PTC were collected and analyzed. Approval to conduct this study was obtained from the Institutional Review Board of West China Hospital of Sichuan University, and written informed consent was obtained from all the study participants. Data were extracted from the electronic medical records and entered into an Excel spreadsheet for analysis. Data collected included the patients' demographics, preoperative ultrasonographic tumor characteristics, surgical details, postoperative pathological details, postoperative outcome, postoperative complications according to the Clavien system, ${ }^{12,13}$ tumor recurrence, and long-term tumor-free survival. Neutrophil-to-lymphocyte ratio (NLR) ${ }^{14,15}$ and platelet-to-lymphocyte ratio (PLR) ${ }^{16}$ that have been reported as predictive factors for tumor recurrence were also included in our analysis and comparison.

All patients underwent preoperative ultrasonography to evaluate the location of the primary tumor, and FNA cytology was conducted to confirm the preoperative diagnosis. Three surgeons with at least 15 years of thyroid and parathyroid surgery experience performed all the operations, and the histopathological evaluation of thyroid specimens was performed by one of two pathologists in our institution. Both thyroid gland and nodal tissue were evaluated from $1 \mathrm{~mm}$-thick anatomic slices. Contralateral level VI dissection was performed mainly depending on the presence of lymph node metastasis in prelaryngeal, orotracheal, or gland invasion. Lateral neck dissection was performed only in cases with clinically evident node metastases in preoperative FNA cytology or intraoperative frozen sections. The surgical protocols were recommended to the patients mainly based on the tumor's diameter, location, capsular invasion, lymph node metastasis, and patients' will; the data of total and subtotal thyroidectomy group are different, attributing not only to the tumor location in isthmus but also to the procedure itself. Postoperative therapy and follow-up included I-131 radioiodine therapy for selected patients, TSH suppression therapy for all patients, and thyroglobulin measurement and ultrasonography to detect tumor recurrence.

The clinical characteristics considered for univariate and multivariate analysis of PTC recurrence in isthmic PTC patients were age at diagnosis ( $\leq 45$ years versus $>45$ years), sex (male versus female), clinical lymph node metastasis at the time of diagnosis ( $\mathrm{cN} 0$ versus $\mathrm{kN}+$ ), postoperative histopathological results ( $\mathrm{cN} 0$ versus $\mathrm{kN}+$ ), $\mathrm{NLR}(\leq 3$ versus $>3$ ), greatest diameter of the tumor $(\leq 10 \mathrm{~mm}$ versus $>10 \mathrm{~mm})$, capsular invasion (yes versus no), coexisting autoimmune thyroid disease (yes versus no), lateral node involvement (yes versus no), and postoperative I-131 radioiodine therapy (yes versus no).

The primary outcome measure was long-term tumorfree survival and postoperative complications. The second outcome measures included parathyroid function recovery (parathyroid hormone and calcium levels on postoperative day 1 and 30 ). 
This retrospective analysis was performed according to an intention-to-treat principle (ITTA) using the preoperative and intraoperative characteristics at the intraoperative decision point of applying either total thyroidectomy or other procedures. The 103 patients with isthmic PTC were subdivided into two groups based on the largest diameter of the isthmic lesion: group A included patients with PTC $\leq 10 \mathrm{~mm}$ (microcarcinoma), and group B included patients with PTC $>10 \mathrm{~mm}$, according to the World Health Organization criteria. ${ }^{8}$

Continuous data were expressed as mean $\pm \mathrm{SD}$, and the differences between continuous data were analyzed using the Mann-Whitney $U$-test. Differences between the categorical data were analyzed using the chi-squared and Fisher's exact test (two-tailed), if necessary. Tumor recurrence outcomes were analyzed using the Kaplan-Meier method. Univariate analyses were performed to identify factors predicting tumorfree survival. All variables with $P<0.05$ were included in the multivariate analysis to assess independent predictive factors using Cox regression analysis. The Cox proportional hazards model was used to generate adjusted hazard ratios (HR) and 95\% confidence intervals (CI). A $P$-value of $<0.05$ was considered statistically significant. SPSS software (SPSS 17; SPSS Inc., Chicago, IL, USA) was used for statistical analysis.

\section{Results}

\section{Patient and tumor characteristics}

A total of 3,068 patients undergoing thyroidectomy due to thyroid cancer in our center were enrolled. Of them, cases of follicular thyroid carcinoma $(\mathrm{n}=116,3.8 \%)$ and medullary thyroid carcinoma $(n=84,2.7 \%)$ were excluded. In addition, most of the PTCs were located in the lobe $(n=2,760$, $90.0 \%$ ), and these patients were also excluded. A total of 108 PTC cases had tumors located at the isthmus; however, two patients were lost to follow-up, and three cases with missing data were also excluded. Thus, 103 PTC cases with tumors located at the isthmus were included in our analysis (as shown in Figure 1). For these patients, five surgical protocols were performed due to the preoperational and intraoperational tumor characteristics and presence of lymph node metastasis. The patients' baseline characteristics are

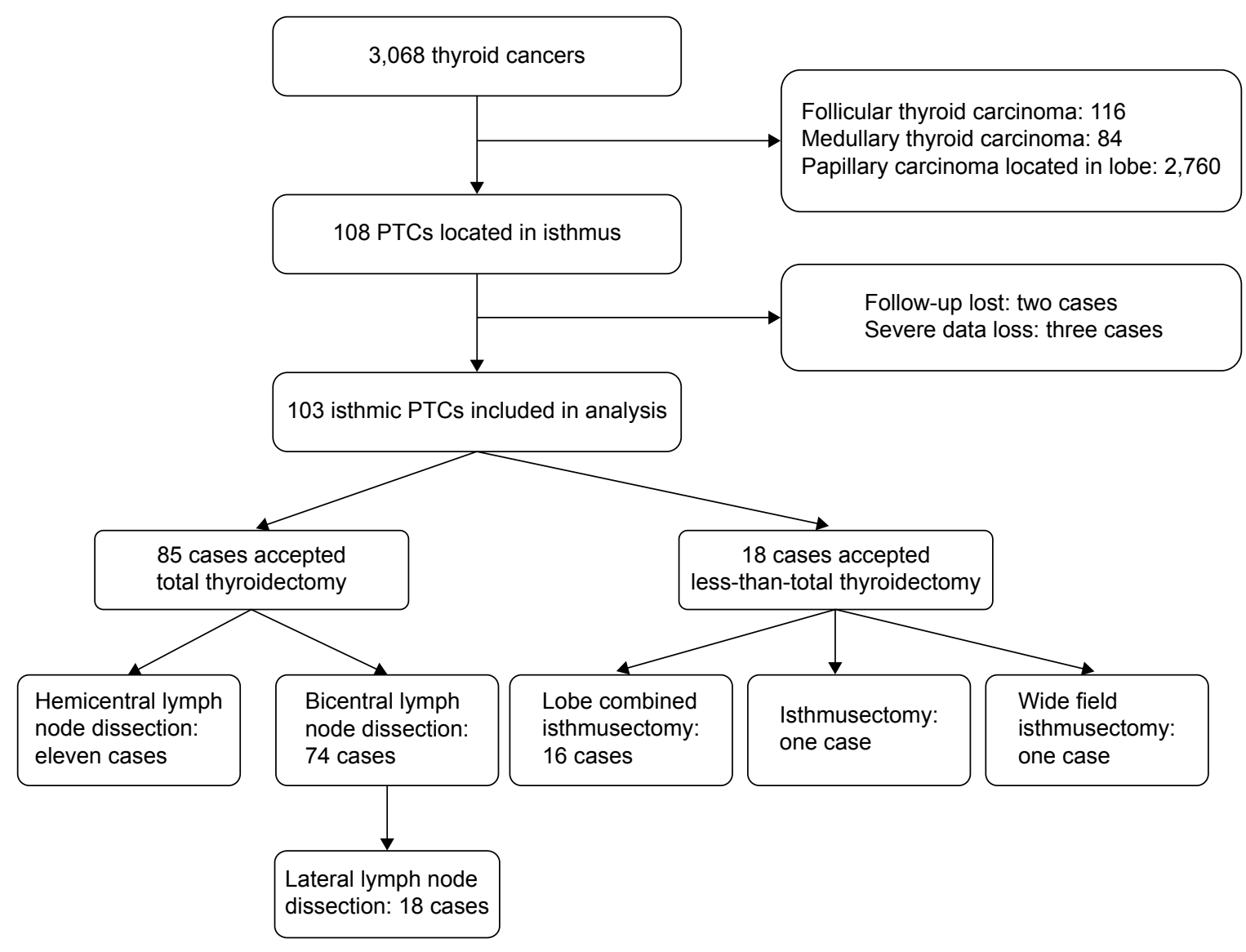

Figure I Synopsis of the study, including isthmic PTC patients with different surgical protocols. Abbreviation: PTC, papillary thyroid cancer. 
Table I Baseline and tumor preoperative features of the isthmic PTC patients

\begin{tabular}{|c|c|c|c|}
\hline \multirow[t]{2}{*}{ Preoperative features } & \multirow{2}{*}{$\begin{array}{l}\text { Total thyroidectomy group } \\
85\end{array}$} & \multirow{2}{*}{$\begin{array}{l}\text { Less-than-total thyroidectomy group } \\
18\end{array}$} & \multirow[t]{2}{*}{$P$-value } \\
\hline & & & \\
\hline Age at diagnosis (mean $\pm S D$, years) & $44.4 \pm 15.4$ & $43.2 \pm 14.6$ & 0.768 \\
\hline$\leq 45$ & 50 & 10 & 0.799 \\
\hline$>45$ & 35 & 8 & \\
\hline Sex (male/female) & $19 / 66$ & $4 / 14$ & 0.990 \\
\hline Height $(\mathrm{cm})$, mean \pm SD & $162.6 \pm 7.5$ & $|56.8 \pm| \mid .2$ & 0.013 \\
\hline Weight $(\mathrm{kg})$, mean \pm SD & $63.1 \pm 13.7$ & $60.8 \pm 10.9$ & 0.505 \\
\hline BMI $\left(\mathrm{kg} / \mathrm{m}^{2}\right)$, mean $\pm \mathrm{SD}$ & $23.1 \pm 4.5$ & $24.9 \pm 7.6$ & 0.208 \\
\hline Multifocality (yes/no) & $24 / 61$ & $4 / 14$ & 0.654 \\
\hline Bilaterality (yes/no) & $3 / 82$ & $0 / 18$ & 0.268 \\
\hline Autoimmune thyroid disease (yes/no) & $22 / 63$ & $2 / 16$ & 0.180 \\
\hline Capsule invasion (yes/no) & $57 / 28$ & $4 / 14$ & $<0.001$ \\
\hline Extrathyroid extension (yes/no) & $3 / 82$ & $0 / 18$ & 0.421 \\
\hline Total tumor size (mean $\pm \mathrm{SD}, \mathrm{mm})$ & $14.4 \pm 8.9$ & $12.2 \pm 10.9$ & 0.373 \\
\hline Total tumor size $(\leq \mathrm{I} \mathrm{cm},>\mathrm{I} \mathrm{mm})$ & $35 / 50$ & $1 \mathrm{I} / 7$ & 0.124 \\
\hline Isthmic tumor size (mean $\pm \mathrm{SD}, \mathrm{mm})$ & $12.4 \pm 8.1$ & $10.2 \pm 9.9$ & 0.306 \\
\hline Isthmic tumor size $(\leq 10 \mathrm{~mm},>10 \mathrm{~mm})$ & $48 / 37$ & $13 / 5$ & 0.219 \\
\hline Largest tumor size (mean $\pm \mathrm{SD}, \mathrm{mm})$ & $13.5 \pm 8.4$ & $10.4 \pm 10.1$ & 0.184 \\
\hline $\mathrm{cN}$ stage $(\mathrm{NO} / \mathrm{NI})$ & $79 / 6$ & $16 / 2$ & 0.561 \\
\hline pN stage (No/NI) & $33 / 52$ & $9 / 9$ & 0.372 \\
\hline pN stage (No/NIb) & $67 / 18$ & $0 / 0$ & $<0.001$ \\
\hline NLR (mean $\pm \mathrm{SD})$ & $2.0 \pm 0.8$ & $2.1 \pm 1.0$ & 0.835 \\
\hline$\leq 3$ & 77 & 16 & 0.826 \\
\hline$>3$ & 8 & 2 & \\
\hline $\operatorname{PLR}($ mean $\pm \mathrm{SD})$ & $117.7 \pm 54.1$ & $103.2 \pm 69.4$ & 0.330 \\
\hline$\leq 200$ & 76 & 17 & 0.514 \\
\hline$>200$ & 9 & 1 & \\
\hline TSH level (mU/L, mean \pm SD) & $5.3 \pm 12.8$ & $2.9 \pm 2.3$ & 0.446 \\
\hline FT4 level $(p m o l / L$, mean \pm SD) & $16.0 \pm 3.3$ & $18.4 \pm 3.6$ & 0.006 \\
\hline FT3 level $($ pmol/L, mean \pm SD) & $5.0 \pm 0.9$ & $4.7 \pm 1.4$ & 0.388 \\
\hline
\end{tabular}

Abbreviations: BMI, body mass index; cN, clinical lymph node metastasis; FT3, free triiodothyronine; FT4, free thyroxine; NLR, neutrophil-to-lymphocyte ratio; PLR, platelet-to-lymphocyte ratio; pN, pathological lymph node metastasis; PTC, papillary thyroid cancer; SD, standard deviation; TSH, thyroid stimulating hormone.

summarized in Table 1. Most of the patients were female (80 cases, 70.8\%). The PTCs in 75 (66.4\%) patients had only one target, and three $(2.9 \%)$ cases showed extrathyroid invasion; the details of the PTC and lymph node characteristics are listed in Table 1. In total, 61 cases (59.2\%) showed pathologically proven lymph node metastasis. Even for the 56 cases with a tumor diameter $\leq 10 \mathrm{~mm}$, capsule invasion was observed in 25 cases (44.6\%), and lymph node characteristics were observed in 28 patients (50\%). Lateral lymph node metastasis was observed in 18 of 103 patients (17.4\%).

Two major groups were established according to whether total thyroidectomy was performed: total thyroidectomy (85 patients) and less-than-total thyroidectomy (18 cases: 16 cases with lobe combined isthmusectomy, one case with isthmusectomy only, one case with isthmus and wide field isthmusectomy). The patients' baseline characteristics and tumor features of the two groups were listed and compared, as shown in Table 1. No significant differences were observed between the two groups except in patient height, the presence of tumor capsule invasion and LT4 level: the patients in the total thyroidectomy group were taller $(P=0.013)$ and included more cases with capsule invasion $(P<0.001)$ and lower free thyroxine (FT4) levels $(P=0.006)$ when compared with the other group. The other details of the comparison are listed in Table 1. The increased capsule invasion in the total thyroidectomy group was mainly due to intraoperative selection bias, as patients with PTC with macroscopic capsule invasion are more likely to accept total thyroidectomy.

\section{Postoperative recovery and surgical- related complications}

According to the Clavien-Dindo system for complication grading, 17 patients of the 103 cases $(16.5 \%)$ suffered postoperative surgical-related complications. Of these, ten patients (9.7\%) suffered grade 1 complications, including postoperative vomit (four cases), pain (three cases), fever (two cases), 
and incision infection (one case); five patients (4.9\%) suffered grade 2 complications, including hoarseness requiring persistent observation and treatment (two cases), severe postoperative hypocalcemia needing treatment (one case), lower extremity deep venous thrombosis (one case), and cervical chylous leakage (one case); and two patients (1.9\%) suffered severe complications (grade 3), including one patient who suffered intractable chylous leakage with the application of Pseudomonas aeruginosa injection and one patient who suffered postoperative bleeding and accepted reoperation. No grade 4 or 5 complications were observed in these 103 cases. When we compared the complication rate of the total thyroidectomy group with that of the less-than-total thyroidectomy group, although the postoperative complication rates were higher in the total thyroidectomy group, with 15 of 85 patients (17.6\%) compared with two of 18 patients $(11.1 \%)$ in the less-than-total thyroidectomy group, this difference did not reach statistical significance $(P=0.500)$. Meanwhile, two patients in the total thyroidectomy group and no patients in the less-than-total thyroidectomy group suffered severe complications, with no significant difference $(P=0.513)$ between the two groups as shown in Table 2 . No recurrent laryngeal nerve injury or permanent palsy occurred.

For comprehensive comparison, we evaluated and compared other recovery factors such as the length of postoperative hospital stay, overall drainage volume, and overall hospitalization costs. No significant differences were observed between the two groups with respect to these three aspects $(P>0.05)$. Meanwhile, we also evaluated parathyroid function recovery; although the total thyroidectomy group showed a much higher rate $(P=0.004)$ and number $(P<0.001)$ of parathyroidectomies, all of these dissected parathyroids were confirmed by intraoperative frozen pathology and were autotransplanted in the sternocleidomastoid. Thus, total thyroidectomy with parathyroid autotransplantation did not significantly damage the function of the parathyroid, with comparable parathyroid hormone and serum calcium levels at the first day and 1 month after surgery $(P>0.05)$.

\section{Intention-to-treat tumor recurrence rate and prognostic factors}

The median follow-up period was 22 months (range 2-54 months). Tumor recurrence was observed in five patients, including two patients in the total thyroidectomy group and three patients in the less-than-total thyroidectomy group. No patient died from tumor recurrence, and most cases of recurrence (four cases, $80 \%$ ) occurred $>30$ months after the operation. An intention-to-treat tumor recurrence comparison was made between the two groups, as shown in Figure 2; the tumor recurrence rate in the total thyroidectomy group was significantly lower than that in the less-than-total thyroidectomy group $(P=0.040)$.

\section{Univariate and multivariate analysis for recurrence}

A total of 16 variables that could be risk factors for PTC recurrence were included in the univariate analysis: age at diagnosis ( $\leq 45 />45$ ), sex (male/female), body mass index ( $\leq 24 />24)$, multifocality (yes/no), bilaterality (yes/no), autoimmune thyroid disease (yes/no), capsule invasion

Table 2 Postoperative recovery of the two patient groups

\begin{tabular}{|c|c|c|c|}
\hline \multirow[t]{2}{*}{ Complications } & \multirow{2}{*}{$\begin{array}{l}\text { Total thyroidectomy group } \\
85\end{array}$} & \multirow{2}{*}{$\begin{array}{l}\text { Less-than-total thyroidectomy group } \\
18\end{array}$} & \multirow[t]{2}{*}{ P-value } \\
\hline & & & \\
\hline Clavien-Dindo classification & $15(17.6 \%)$ & $2(11.1 \%)$ & 0.500 \\
\hline Grade I & $10(11.8 \%)$ & 0 & \\
\hline Grade II & $3(3.5 \%)$ & $2(11.1 \%)$ & \\
\hline Grade IIla & $\mathrm{I}(\mathrm{l} .2 \%)$ & 0 & \\
\hline Grade IIIb & $\mathrm{I}(\mathrm{I} .2 \%)$ & 0 & \\
\hline Total hospital stay days, mean \pm SD & $5.9 \pm 2.1$ & $7.3 \pm 5.2$ & 0.070 \\
\hline Postoperative drainage volume $(\mathrm{mL})$, mean $\pm \mathrm{SD}$ & $179.1 \pm 212.7$ & $198.7 \pm 164.4$ & 0.713 \\
\hline Overall hospitalization costs (dollars), mean \pm SD & $3,345.4 \pm 878.5$ & $3,235.7 \pm 924.3$ & 0.635 \\
\hline Parathyroids autologous transplantation (yes/no) & $4 I / 44$ & $2 / 16$ & 0.004 \\
\hline Parathyroids autologous transplantation number & $0.7 \pm 0.8$ & $0.1 \pm 0.3$ & $<0.001$ \\
\hline PTH level (pmol/L, the first day) & $2.2 \pm 1.6$ & $2.6 \pm 1.7$ & 0.337 \\
\hline Serum calcium level (mmol/L, the first day) & $2.1 \pm 0.2$ & $2.0 \pm 0.1$ & 0.651 \\
\hline PTH level (pmol/L, I month) & $3.7 \pm 2.0$ & $3.6 \pm 1.7$ & 0.878 \\
\hline Serum calcium level (mmol/L, I month) & $2.3 \pm 0.7$ & $2.2 \pm 0.1$ & 0.753 \\
\hline
\end{tabular}

Abbreviation: PTH, parathyroid hormone. 


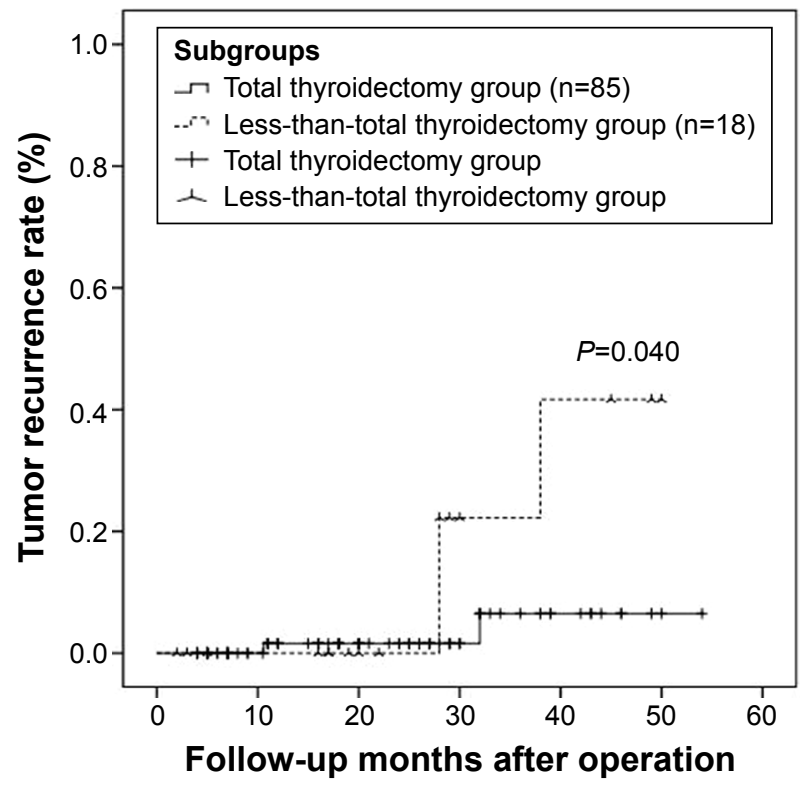

Figure 2 Unadjusted comparison of the intention-to-treat recurrence for patients with isthmic PTCs receiving either total thyroidectomy or less-than-total thyroidectomy.

Abbreviation: PTC, papillary thyroid cancer.

(yes/no), extrathyroid extension (yes/no), total tumor size $(\leq 1 \mathrm{~cm} />1 \mathrm{~cm})$, isthmic tumor size $(\leq 10 \mathrm{~mm} />10 \mathrm{~mm})$, $\mathrm{cN}$ stage $(\mathrm{N} 0 / \mathrm{N} 1), \mathrm{pN}$ stage $(\mathrm{N} 0 / \mathrm{N} 1), \mathrm{NLR}(\leq 3 />3)$, PLR ( $\leq 200 />200$ ), TSH level (low/normal), and surgical protocol (total thyroidectomy/less-than-total thyroidectomy). Univariate analysis identified the following factors as predicting tumor recurrence: $\mathrm{pN}$ stage $\mathrm{N} 1(P=0.031)$, PLR $>200(P=0.028)$, and less-than-total thyroidectomy $(P<0.001)$. Multivariate analysis identified less-than-total thyroidectomy as a risk factor for tumor recurrence in PTC cases with tumors located at the isthmus (HR: 1.870, 95\% CI: $1.320-2.218, P<0.001)$.

\section{Discussion}

The thyroid isthmus is the central part of the thyroid gland and lies anterior to the trachea, overlying the second to fourth tracheal rings and being covered by the strap muscles, fascia, and skin in the middle of the neck. ${ }^{8}$ The rate of differentiated carcinoma located in the isthmus was reported to range from $1 \%$ to $9.2 \%$ for all thyroid malignant carcinoma nodules. ${ }^{9} 10$ In our present study, the rate of patients with dominant nodules of the thyroid isthmus was 3.9\% among PTCs, which is comparable with that of previous reports. Thus, a dominant thyroid nodule of the isthmus is an uncommon lesion and requires surgery. For PTCs, despite excellent outcomes, with a lower risk of death or disease recurrence (indolent tumor) than patients with most other malignancies, mortality rates may be increasing in some subgroups,${ }^{17}$ and the loss of quality of life should also demand our attention. ${ }^{18}$ Until now, the extent of appropriate surgical resection for PTCs located in the isthmus has remained a matter of considerable debate; furthermore, no consensus exists in the guidelines for the surgical protocol of PTCs confined to the isthmus. Most previous studies support total thyroidectomy as the standard surgical protocol for PTCs located in the isthmus. ${ }^{8-10}$ However, Nixon et $\mathrm{al}^{10}$ reported that cases with PTC nodules confined to the isthmus without evidence of extraglandular extension may be suitable for thyroid isthmusectomy or wide field isthmusectomy due to the reduced chance of damaging the recurrent laryngeal nerve and parathyroid glands without exposing the tracheoesophageal grooves. However, only six patients treated with isthmusectomy were reported in this study, which may limit the argument for choosing isthmusectomy as the standard surgical protocol for isthmic PTCs. We also performed one isthmusectomy and one wide field isthmusectomy for isthmic PTCs; however, the lack of follow-up beyond 5 months may limit our ability to draw conclusions. In our present study, an overall pathological incidence of lymph node metastasis of 59.2\% (61 cases) was observed in patients with PTC in the isthmus. Even for the 56 cases with tumor diameters $\leq 10 \mathrm{~mm}$, capsule invasion was observed in 25 cases (44.6\%), and lymph node characteristics were observed in 28 patients $(50 \%)$. The rate of lymph node metastasis in our results was much higher than that reported in other studies. ${ }^{8,9,19}$ The main reason for this higher rate was that we defined lymph node metastasis as any node, including the central (including prelaryngeal and pretracheal) and lateral compartments, showing metastases. In previous studies, the lymph node metastasis rate of patients with PTC located in the isthmus versus the lobes was compared, and the former location showed a much higher rate of lymph node metastasis. ${ }^{8}$ There are some potential reasons for this higher lymph node metastasis rate. First, with regard to the anatomical location, the isthmus is a very thin piece of thyroid tissue, and even tumors with a small diameter can very easily undergo capsular invasion. ${ }^{8}$ Lymphatic drainage of the isthmus may also contribute to the higher lymph node metastasis rate, as lymph vessels from the isthmus may drain into the pretracheal, prelaryngeal, and bilateral central compartment lymph nodes. Meanwhile, lateral node involvement was also commonly observed in our study at $17.5 \%$, which is comparable with other previous studies. ${ }^{9} 19$ Lymphatic channels of the thyroid gland develop more richly posterolaterally than anteromedially, 
which may have contributed to the higher rate of lateral lymph node metastasis.

PTCs arising in the isthmus are more likely to have multiple foci, capsular invasion, and invasion of adjacent tissues such as the trachea and strap muscles, even with a smaller tumor size, when compared with PTCs located in the lobe. ${ }^{20,21}$ The multifocality seems to be related to the midline position of the tumors, which easily spread to the bilobes, and more frequent capsular invasion appears to result from tumors growing in the thin thyroid isthmus. ${ }^{9}$ In our present study, the multifocality rate was $27.2 \%$, which is lower than that in other reports. This difference may be explained by evaluation bias, as we defined multifocality as involving two or more pathologically proven PTCs. These risk factors may contribute to the high risk of recurrence after surgery.

Because the surgical protocols for these high-risk recurrence isthmic PTCs are critically important, our present study evaluated the tumor characteristics of isthmic PTCs and compared the outcomes, including tumor recurrence, postoperative complications, and parathyroid recovery, between total thyroidectomy and less-than-total thyroidectomy groups. The total thyroidectomy group showed a significantly lower tumor recurrence rate, and more radical thyroidectomy may contribute to the advantage of total thyroidectomy. Lymph system metastasis is the most common metastasis pathway, and in the thyroid gland, the lymph duct system is very rich. ${ }^{22,23}$ As a result, intragland metastasis is very common for PTCs, as demonstrated by the multifocality shown in our results. Therefore, some cellular metastatic foci may be residual if less-than-total thyroidectomy is performed.

The safety of the surgical protocol is also a very important aspect for group comparison. We compared the postoperative complications according to the Clavien-Dindo system and found no difference between the two groups, indicating that total thyroidectomy is relatively safe when compared with less-than-total thyroidectomy. No recurrent laryngeal nerve injury or permanent palsy occurred in the total thyroidectomy group. Experienced surgeons and intraoperational real-time monitoring may contribute to this advantage. ${ }^{24}$ The postoperative recovery factors of the two groups were also evaluated and compared. The most important point for this comparison was parathyroid function recovery; although the total thyroidectomy group showed many parathyroidectomies, all of these removed parathyroids were confirmed by intraoperative frozen pathology and were autotransplanted in the sternocleidomastoid, with the surgical details presented in our previous research. ${ }^{25}$ Carbon nanoparticles as a lymph node tracer may help to identify affected lymph nodes and preserve the parathyroid. ${ }^{26,27}$

There are several limitations to this study. First, the main limitation is related to its single-center analysis and the limited series of isthmic PTC patients. Second, the study only made comparisons among patients with isthmic PTCs, without comparing PTCs located in the isthmus and the lobe, as in other reports. Third, the mean follow-up time of 22 months may have limited our analysis. Thus, larger randomized multicenter studies are needed to confirm our results.

\section{Conclusion}

Due to multiple foci, capsular invasion, and lymph node metastasis in isthmic PTCs, total thyroidectomy seems to be more appropriate than less-than-total thyroidectomy because of its comparable postoperational complications and recovery but reduced recurrence.

\section{Acknowledgment}

Lei Jianyong and Zhong Jinjing are joint first authors of this article.

\section{Disclosure}

No benefits in any form have been received or will be received from a commercial party related directly or indirectly to the subject of this article. The authors report no other conflicts of interest in this work.

\section{References}

1. Siegel R, Naishadham D, Jemal A. Cancer statistics, 2012. CA Cancer J Clin. 2012;62(1):10-29.

2. Chen AY, Jemal A, Ward EM. Increasing incidence of differentiated thyroid cancer in the United States, 1988-2005. Cancer. 2009; 115(16):3801-3807.

3. Lee J, Seol MY, Jeong S, et al. A metabolic phenotype based on mitochondrial ribosomal protein expression as a predictor of lymph node metastasis in papillary thyroid carcinoma. Medicine (Baltimore). 2015;94(2):e380.

4. Mihailescu DV, Schneider AB. Size, number, and distribution of thyroid nodules and the risk of malignancy in radiation-exposed patients who underwent surgery. J Clin Endocrinol Metab. 2008;93(6): 2188-2193.

5. Frates MC, Benson CB, Charboneau JW, et al. Management of thyroid nodules detected at US: society of radiologists in ultrasound consensus conference statement. Radiology. 2005;237(3):794-800.

6. Davies L, Welch HG. Increasing incidence of thyroid cancer in the United States, 1973-2002. JAMA. 2006;295(18):2164-2167.

7. American Thyroid Association (ATA) Guidelines Taskforce on Thyroid Nodules and Differentiated Thyroid Cancer, Cooper DS, Doherty GM, et al. Revised American Thyroid Association management guidelines for patients with thyroid nodules and differentiated thyroid cancer. Thyroid. 2009;19(11):1167-1214.

8. Karatzas T, Charitoudis G, Vasileiadis D, Kapetanakis S, Vasileiadis I. Surgical treatment for dominant malignant nodules of the isthmus of the thyroid gland: a case control study. Int J Surg. 2015;18:64-68. 
9. Lee YS, Jeong JJ, Nam KH, Chung WY, Chang HS, Park CS. Papillary carcinoma located in the thyroid isthmus. World J Surg. 2010;34(1): 36-39.

10. Nixon IJ, Palmer FL, Whitcher MM, et al. Thyroid isthmusectomy for well-differentiated thyroid cancer. Ann Surg Oncol. 2011;18(3): $767-770$.

11. Watkinson JC, British Thyroid A. The British Thyroid Association guidelines for the management of thyroid cancer in adults. $\mathrm{Nucl} \mathrm{Med}$ Commun. 2004;25(9):897-900.

12. Vonlanthen R, Slankamenac K, Breitenstein S, et al. The impact of complications on costs of major surgical procedures: a cost analysis of 1200 patients. Ann Surg. 2011;254(6):907-913.

13. Clavien PA, Barkun J, de Oliveira ML, et al. The Clavien-Dindo classification of surgical complications: five-year experience. Ann Surg. 2009; 250(2):187-196.

14. Lang BH, Ng CP, Au KB, Wong KP, Wong KK, Wan KY. Does preoperative neutrophil lymphocyte ratio predict risk of recurrence and occult central nodal metastasis in papillary thyroid carcinoma? World J Surg. 2014;38(10):2605-2612.

15. Kim JY, Park T, Jeong SH, et al. Prognostic importance of baseline neutrophil to lymphocyte ratio in patients with advanced papillary thyroid carcinomas. Endocrine. 2014;46(3):526-531.

16. Kim SM, Kim EH, Kim BH, et al. Association of the preoperative neutrophil-to-lymphocyte count ratio and platelet-to-lymphocyte count ratio with clinicopathological characteristics in patients with papillary thyroid cancer. Endocrinol Metab (Seoul). 2015;30(4):494-501.

17. Mazzaferri EL. Managing small thyroid cancers. JAMA. 2006;295(18): 2179-2182.

18. Duan H, Gamper E, Becherer A, Hoffmann M. Quality of life aspects in the management of thyroid cancer. Oral Oncol. 2015;51(6):S1-S5.

19. Goldfarb M, Rodgers SS, Lew JI. Appropriate surgical procedure for dominant thyroid nodules of the isthmus $1 \mathrm{~cm}$ or larger. Arch Surg. 2012;147(9):881-884.
20. Sugenoya A, Shingu K, Kobayashi S, et al. Surgical strategies for differentiated carcinoma of the thyroid isthmus. Head Neck. 1993;15(2): 158-160.

21. Chen P, Sun R, Pu Y, et al. Pri-Mir-34b/C and Tp-53 polymorphisms are associated with the susceptibility of papillary thyroid carcinoma: a case-control study. Medicine (Baltimore). 2015;94(38):e1536.

22. Tevis SE, Chen H. Papillary thyroid cancer - how aggressive should surgery be? Nat Rev Endocrinol. 2015;11(4):195-196.

23. Carling T, Udelsman R. Thyroid cancer. Annu Rev Med. 2014;65: $125-137$.

24. Gong RX, Luo SH, Gong YP, et al. Prediction of nonrecurrent laryngeal nerve before thyroid surgery - experience with 1825 cases. J Surg Res. 2014;189(1):75-80.

25. Wei T, Li Z, Jin J, et al. Autotransplantation of Inferior Parathyroid glands during central neck dissection for papillary thyroid carcinoma: a retrospective cohort study. Int J Surg. 2014;12(12):1286-1290.

26. Wang B, Qiu NC, Zhang W, et al. The role of carbon nanoparticles in identifying lymph nodes and preserving parathyroid in total endoscopic surgery of thyroid carcinoma. Surg Endosc. 2015;29(10):2914-2920.

27. Li Y, Jian WH, Guo ZM, Li QL, Lin SJ, Huang HY. A meta-analysis of carbon nanoparticles for identifying lymph nodes and protecting parathyroid glands during surgery. Otolaryngol Head Neck Surg. 2015; 152(6):1007-1016.
OncoTargets and Therapy

\section{Publish your work in this journal}

OncoTargets and Therapy is an international, peer-reviewed, open access journal focusing on the pathological basis of all cancers, potential targets for therapy and treatment protocols employed to improve the management of cancer patients. The journal also focuses on the impact of management programs and new therapeutic agents and protocols on

\section{Dovepress}

patient perspectives such as quality of life, adherence and satisfaction The manuscript management system is completely online and includes a very quick and fair peer-review system, which is all easy to use. Visit http://www.dovepress.com/testimonials.php to read real quotes from published authors. 